\title{
Vasoactive-inotropic Score for Early Detection and Mortality Prediction of Sepsis in Children
}

\author{
Aileen Clarissa Dauhan*, Aridamuriany Dwiputri Lubis, Munar Lubis \\ Department of Child Health, Faculty of Medicine, Universitas Sumatera Utara, \\ J1. Dr. Mansyur No.5 Padang Bulan, Medan Baru, Medan, Indonesia \\ *Corresponding author. E-mail: aileen.clarissa.dauhan@gmail.com
}

Received date: Jun 28, 2020; Revised date: Oct 19, 2020; Accepted date: Oct 30, 2020

\section{Abstract}

B ACKGROUND: Early detection and treatment of sepsis can prevent septic shock and reduce mortality rate. Troponin can become a prognostic factor in sepsis. However, not all health facilities are equipped to assess troponin levels. Vasoactive-inotropic score (VIS) is a simpler and more accessible method to describe hemodynamic status. The aim of this study was to assess the suitability of VIS score as early prognosis and mortality predictor of sepsis

METHODS: A retrospective study was conducted to determine the correlation between VIS and troponin levels for sepsis cases in Pediatric Intensive Care Unity (PICU) Haji Adam Malik Hospital, Medan from January 2018 to December 2019. VIS score at 48 hours, maximum VIS score, pediatric logistic organ dysfunction-2 (PELOD-2) score, cardiac troponin levels at 48 hours were taken from medical records.

\section{Introduction}

Sepsis is a life-threatening condition with organ dysfunction due to dysregulated immune as a response to infections. (1) Untreated sepsis can progress to septic shock which results in multiple organ failures such as the brain, kidneys and cardiovascular system. The cardiovascular system is the most susceptible to sepsis condition. Mortality rate increases from $70 \%$ to $90 \%$ with the presence of myocardial dysfunction in children with sepsis. $(2,3)$
RESULTS: There were 54 samples analyzed. VIS scores were positively correlated $(p<0.001)$ to troponin $\mathrm{T}$ and troponin I levels at 48 hours $(\mathrm{r}=0.670$ and $\mathrm{r}=0.606$, respectively). VIS at 48 hours and maximum VIS were related to mortality ( $p=0.001$ and $p<0.001$, respectively). VIS score at 48 hours could be used as a predictive factor for mortality (area under the curve (AUC): 79.7\%, $p<0.001$ ) with a cut-off point at $11(74.4 \%$ sensitivity and $80 \%$ specificity). High VIS at 48 hours indicated poor outcomes of sepsis in children with odd ratio (OR) value: 1.99 (95\% confidence interval (CI): 1.25-3.19).

CONCLUSION: Vasoactive-inotropic score was suitable as an alternative to cardiac troponin $\mathrm{T}$ and troponin I levels at 48 hours to early detect myocardial dysfunctions and mortality in children.

KEYWORDS: troponin, vasoactive-inotropic score, sepsis, children, mortality

Indones Biomed J. 2021; 13(1): 34-9
Hemodynamic monitoring is important in diagnosing and treating sepsis. Both invasive and non-invasive monitoring methods can be used in hemodynamic and myocardial dysfunction monitoring in sepsis. For instance, cardiac output ( $\mathrm{CO}$ ) and volume status monitoring by echocardiography or transesophageal doppler, monitoring of central venous pressure (CVP), measurement of invasive arterial pressure and ultrasonic cardiac output monitor (USCOM).(4) Echocardiography is most often used to diagnose impaired cardiac function. This method, however, requires accurate subjective skills of trained experts and 
there are difficulties to provide radiographic modalities within 24 hours.(5) Therefore, biomarkers and scoring for myocardial dysfunction are required to provide information on the management and prognostic evaluation of sepsis in children.

The stress occurs from sepsis can increase oxygen requirement in the myocardium and stimulate troponin release. $(5,6)$ Troponin is not found in the extracellular, therefore, its presence in the blood is a sensitive and specific marker of myocardial dysfunction to evaluate and assess severe sepsis and septic shock.(6) The increase in troponin levels in sepsis is associated with poor prognosis, severe sepsis condition and structural abnormalities in cardiac muscle cells. The value of troponin can be a predictive factor for mortality of pediatric patients with sepsis, in which the best results are measurable after 48 hours of sepsis diagnosis.(7)

Sepsis and septic shock can be characterized by the inability of the cardiovascular system to meet metabolic needs. Unlike adults, children have diverse hemodynamic status. They generally respond to inotropic and vasopressor therapy, which indicates myocardial dysfunction in acute phase of sepsis.(8) Vasoactive and inotropic drugs are standard therapies for cardiovascular dysfunction and hypotension that occur in children with septic shock.(9) Besides troponin levels, vasoactive-inotropic score (VIS) can also be a marker to assess cardiovascular involvement in children with sepsis.(9) It can become an early prognostic indicator of sepsis in children at the intensive care units.

Vasoactive-inotropic score analysis is a simpler and more straightforward hemodynamic observations parameter than troponin levels. This becomes an advantage for pediatric intensive care units with limited facilities to early detect and treat sepsis. The need to assess the relationship and significance between troponin levels and VIS is to increase the confidence level to substitute troponin for VIS assessment. Moreover, how VIS score predicts the mortality of sepsis in children need to be observed.

This research was conducted to assess the suitability of VIS score as an alternative to Troponin (troponin T, troponin I) levels for sepsis prognosis and its severity, as well as mortality predictor in children.

\section{Methods}

Retrospective study was conducted to determine the correlation between VIS and troponin level (troponin $\mathrm{T}$ and troponin I) in children with sepsis at 48 hours admission to
Pediatric Intensive Care Unity (PICU) of Haji Adam Malik Hospital, Medan. Medical records of children with sepsis diagnosis and vasoactive inotropic drug administration within 48 hours of treatment from January 2018 to December 2019 were taken. Children with heart disease were excluded in this research. This study was approved by Health Research Ethical Committee Faculty of Medicine, Universitas Sumatera Utara/Haji Adam Malik General Hospital (No. 884/TGL/KEPK FK USU-RSUP HAM/2019). Data, such as age, gender, primary organ involvement, nutritional status and treatment outcome (survival or mortality), was collected from the medical records of subjects who met the research criteria.

\section{Troponin Level Assessment}

Troponin $\mathrm{T}$ and troponin I values of research subjects were assessed. Troponin $\mathrm{T}$ values were obtained from the examination done in the integrated laboratory of Faculty of Medicine, Universitas Sumatera Utara. About $2 \mathrm{~mL}$ of venous blood samples were prepared in heparin tube and centrifuged for 10 minutes at $3000 \mathrm{rpm}$ speed. Then the sample was stored in $-80^{\circ} \mathrm{C}$ and kept stable for 6 months period. Troponin $\mathrm{T}$ examination was done by using a double antibody sandwich enzyme-linked immunosorbent one-step process assay (ELISA) (Catalog No. QY-E03068, QayeeBio, Shanghai, China). The result was recorded in $\mathrm{ng} / \mathrm{mL}$ unit with assay ranging from $7.8-500 \mathrm{ng} / \mathrm{mL}$.

Troponin I values were obtained from the examination done by using RAMP ${ }^{\circledR}$ Troponin test (Catalog No. C1101, Response Biomedical, Vancouver, BC, Canada) in the laboratory of Haji Adam Malik Hospital. Two $\mathrm{mL}$ ethylenediaminetetraacetic acid (EDTA) whole blood samples were taken and analyzed immediately by using cartridges in the test reader. The assay was immunochromatographic test that quantitatively measure the levels of Troponin I within 19 minutes. The result for Troponin I was recorded in $\mathrm{ng} / \mathrm{mL}$ unit with reportable range of $0-32 \mathrm{ng} / \mathrm{mL}$. The results exceeding $32 \mathrm{ng} / \mathrm{mL}$ were reported as $>32 \mathrm{ng} / \mathrm{mL}$.

\section{Vasoactive-inotropic Score (VIS) Calculation Method}

The requirement doses of inotropic and vasopressor therapy was recorded as VIS that validated the amount of support the cardiovascular system needs. The doses were recorded at the first 48 hours after the diagnosis of sepsis in PICU. The calculation was in dosage/ $\mathrm{kg}$ body weight, with the formula as follow. $(9,10)$

VIS is calculated by accumulating dopamine dose $(\mu \mathrm{g} /$ $\mathrm{kg} / \mathrm{min})$, dobutamine dose $(\mu \mathrm{g} / \mathrm{kg} / \mathrm{min}), 100 \mathrm{x}$ adrenaline 
dose $(\mu \mathrm{g} / \mathrm{kg} / \mathrm{min}), 100 \times$ noradrenaline dose $(\mu \mathrm{g} / \mathrm{kg} / \mathrm{min})$, $10 \mathrm{x}$ milrinone dose $(\mu \mathrm{g} / \mathrm{kg} / \mathrm{min})$, and $10.000 \mathrm{x}$ vasopressin dose $(\mathrm{U} / \mathrm{kg} / \mathrm{min})$.

\section{Statistical Analysis}

Statistical calculation was done at $95 \%$ confidence interval where $p<0.05$ was considered as significant. A diagnostic test was done to examine the cut-off, receiver operator curve (ROC), areas under the curve (AUC) and odds ratio (OR) of VIS value at 48 hours to predict the mortality of sepsis cases in children. Statistical analysis was performed with SPSS version 22.0 program (IBM Coorporation, Armonk, NY, USA) for Windows.

\section{Results}

\section{Basic Characteristic Data of Research Sample}

There were 54 medical records that met the inclusion criteria from January 2018 to December 2019 period. The number of male and female subjects was equal at 27 subjects for each gender. The median age was 1 year and 8 months, with age range from one month old to 17 years old. The highest primary infection observed in PICU of Haji Adam Malik Hospital was central nervous system disorders, including meningitis and status epilepticus and followed by respiratory disorders. Pediatric logistic organ dysfunction score-2 (PELOD-2) was ranging from 7 to 21 in the first 24 hours. After 48 hours of evaluation, the score was between 3 and 24, which indicated both improved and worsened condition. From the data, there were 15 subjects $(27.8 \%)$ who were moved from PICU, while 39 subjects (72.2\%) died. The characteristic data from the research sample is presented in Table 1.

\section{Vasoactive-inotropic Drug Administration Profile}

In this study, all patients received vasoactive-inotropic drugs in the first 48 hours, such as dopamine in 51 patients (94.4\%), dobutamine in 37 patients (68.5\%), norepinephrine in 12 patients $(22.2 \%)$ and epinephrine in 6 patients $(11.1 \%)$. Some patients received combinations of vasoactive-inotropic drugs and none of the patients were given milrinone or vasopressin. The median value of VIS in the first 48 hours was 15 (ranged between 5 and 50). Over the course of the disease, the need of vasoactive-inotropic drugs increased, where the highest median VIS in patients was at 25 (ranged between 5 and 55). Table 2 shows the profile of supportive therapies together with vasoactiveinotropic score.

\section{The Correlation of Troponin Levels with VIS Value}

Troponin $\mathrm{T}$ and Troponin I showed significant relationship with VIS score at 48 hours of treatment. The correlation was analyzed by using Spearman correlation at 48 hours, with $\mathrm{r}=0.670$ between troponin $\mathrm{T}$ and VIS $(p<0.001)$ and $\mathrm{r}=0.606$ between troponin I and VIS $(p<0.001)$. The data is presented in Table 3, where the higher troponin $\mathrm{T}$ and troponin I levels were, the higher vasoactive-inotropic score (VIS) was recorded.

\section{VIS and Mortality}

Vasoactive-inotropic scores at 48 hours after admission to PICU and the peak obtained during the treatment and observation have an important relationship with mortality. VIS values at 48 hours and the peak of non-survivors were higher than survived children (Table 4).

The ROC curve in Figure 1 gave $79.7 \%(p=0.001)$ AUC. This shows that VIS score at 48 hours can become an indicator to predict mortality in children with sepsis. The cut-off point was measurable at score 11 , with $74.4 \%$ sensitivity and $80 \%$ specificity. The odds ratio (OR) was at

Table 1. Basic characteristics of research subject.

\begin{tabular}{lc}
\hline \multicolumn{1}{c}{ Indicator } & $\mathbf{n}=\mathbf{5 4}$ \\
\hline Gender, (n (\%)) & $27(50)$ \\
Male & $27(50)$ \\
Female & $1.6(0.08-17)$ \\
\hline Age (year), median (min-max) & $9(3-55)$ \\
\hline Bodyweight (kg), median (min-max) & $75.5(43-165)$ \\
\hline Body height (cm), median (min-max) & \\
\hline Nutritional status (n (\%)) & $10(18.5)$ \\
Severe malnutrition & $9(16.7)$ \\
Mild-moderate malnutrition & $34(63)$ \\
Well-nourished & $1(1.9)$ \\
Overweight & $15(27.8)$ \\
\hline PICU indication (n (\%)) & $30(55.5)$ \\
Respiratory disorder & $5(9.3)$ \\
Neurological disorder & $4(7.4)$ \\
Infection and tropical disease & \\
Nephrology disorder & $9(7-21)$ \\
\hline PELOD-2 score, median (min-max) & $10(3-24)$ \\
24 hours & $15(27.8)$ \\
48 hours & $39(72.2)$ \\
\hline Outcome, (n (\%)) & $29(53.7)$ \\
Survival & \\
Death & \\
\hline Culture result (n (\%)) & \\
Positive & \\
Negative & \\
\hline
\end{tabular}


Table 2. Supportive therapies and VIS.

\begin{tabular}{lc}
\hline \multicolumn{1}{c}{ Therapy } & Median (Min-Max) \\
\hline Vasoactive inotropic therapies in 48 hours & \\
Dopamine $(\mu \mathrm{g} / \mathrm{kg} / \mathrm{min}), \mathrm{n}=51$ & $10(5-10)$ \\
Dobutamine $(\mu \mathrm{g} / \mathrm{kg} / \mathrm{min}), \mathrm{n}=37$ & $10(5-10)$ \\
Noradrenaline $(\mu \mathrm{g} / \mathrm{kg} / \mathrm{min}), \mathrm{n}=12$ & $0.05(0.05-0.2)$ \\
Adrenaline $(\mu \mathrm{g} / \mathrm{kg} / \mathrm{min}), \mathrm{n}=6$ & $0.09(0.05-0.3)$ \\
\hline VIS at 48 hours & $15(5-50)$ \\
\hline Peak of vasoactive inotropic therapies & $10(5-15)$ \\
Dopamine $(\mu \mathrm{g} / \mathrm{kg} / \mathrm{min}), \mathrm{n}=52$ & $10(5-15)$ \\
Dobutamine $(\mu \mathrm{g} / \mathrm{kg} / \mathrm{min}), \mathrm{n}=40$ & $0.08(0.05-0.3)$ \\
Noradrenaline $(\mu \mathrm{g} / \mathrm{kg} / \mathrm{min}), \mathrm{n}=19$ & $0.2(0.05-0.3)$ \\
Adrenaline $(\mu \mathrm{g} / \mathrm{kg} / \mathrm{min}), \mathrm{n}=18$ & $25(5-55)$ \\
\hline Peak VIS
\end{tabular}

1.99 (95\% confidence interval (CI): 1.25 - 3.19). This shows that patients with VIS score $\geq 11$ at 48 hours is 1.99 times more at risk of mortality than patents with VIS score $<11$.

\section{Discussion}

A large-scale study conducted worldwide found that the death rate due to sepsis in children was at 25\%.(11) In 2017, the study conducted in PICU of Haji Adam Malik Hospital reported $61 \%$ death case.(7) This study, conducted in the same location, however, reported a higher death rate at $72.2 \%$. Thus, death rate due to sepsis still shows an upward trend.

Fluid resuscitation is the first step of early goaldirected therapy (EGDT) to treat children with sepsis or septic shock. In case of non-responsiveness towards fluid, vasoactive and inotropic drugs administration is the next step to be taken. EGDT steps can reduce mortality rate by one third of the total cases.(8) Vasoconstrictors, such as dopamine and norepinephrine are widely used in the initial phase of septic shock. In this study, dopamine was used the most in the first 48 hours, where $94.4 \%$ patients received the vasoactive drug with a median dose of $10 \mathrm{mcg} / \mathrm{kg} / \mathrm{minute}$ (ranged between 5 and $10 \mathrm{mcg} / \mathrm{kg} /$ minute). Dobutamine was the second highest with $68.5 \%$ patients receiving the drug, followed by norepinephrine and epinephrine with $22.2 \%$ and $11.1 \%$ patients receiving the drugs respectively. All vasoactive and inotropic drugs are important in the management of sepsis with cardiovascular system involvement. The need for drugs varies according to the hemodynamics of patients. The pattern of vasoactive drugs administration indicated cardiovascular system disorders with the requirement to increase cardiac pumps.

The required dosage of vasoactive and inotropic drugs during treatment is measured by vasoactive-inotropic score (VIS).(10) Previous studies stated that VIS value at 48 hours indicated conditions which were strongly associated with myocardial dysfunction and poor health outcomes, hence the need of supports for severely ill conditions.(9) In this study, the median value of VIS in the first 48 hours was 15 (ranged between 5 and 50). Over the course of the disease, the need for vasoactive-inotropic drugs increased with the rise of the median value to 25 (ranged between 5 and 55).

There are physiological and metabolic changes in sepsis, as well as coronary blood flow impairment, decrease in oxygen levels, myocardial hypokinesis and ventricular dilatation.(12) The interruption in blood flow results in the decrease in heart muscle perfusion and leads to myocardial dysfunction and troponin secretion from cardiac myocytes. $(13,14)$

Myocardial dysfunction is common in patients with septic shock. The condition is associated with higher risk of mortality, but it is reversible within 7-10 days. Conventional myocardial dysfunction can be detected by echocardiography, but its utilization is still limited. The presence of sensitive and specific biomarkers for myocardial dysfunction can help to provide prognosis and evaluation to manage and treat sepsis. Early detection of myocardial dysfunction and followed by immediate treatment with inotropic drugs can improve the health outcome and reduce mortality.(15)

VIS value at 48 hours was observed to have strong positive correlations $(p<0.001)$ with troponin $\mathrm{T}$ and

Table 3. Correlation of VIS and cardiac troponin in 48 hours.

\begin{tabular}{lccc}
\hline & Median (Min-Max) & $\mathbf{r}$ & $\boldsymbol{p}^{*}$ \\
\hline VIS & $15(5-50)$ & & \\
Troponin T levels $(\mathrm{ng} / \mathrm{mL})$ & $42.5(10-233)$ & 0.67 & $<0.001$ \\
Troponin I levels $(\mathrm{ng} / \mathrm{mL})$ & $0.7(0-3.11)$ & 0.606 & $<0.001$ \\
\hline
\end{tabular}

*Spearman correlation. 
Table 4. The relationship of VIS and mortality.

\begin{tabular}{lccc}
\hline & $\begin{array}{c}\text { Survivor } \\
\text { Median (min-max) }\end{array}$ & $\begin{array}{c}\text { Non-survivor } \\
\text { Median (min-max) }\end{array}$ & $\boldsymbol{p}^{*}$ \\
\hline VIS at 48 hours & $9(5-20)$ & $20(5-50)$ & 0.001 \\
Peak VIS & $10(5-20)$ & $31(5-55)$ & $<0.001$ \\
\hline
\end{tabular}

*Mann-Whitney test.

troponin I values, with $\mathrm{r}=0.670$ and $\mathrm{r}=0.606$, respectively. The higher the troponin $\mathrm{T}$ and troponin I values at 48 hours, the higher vasoactive-inotropic score obtained. This showed that the increase in troponin levels and VIS score corresponded to the increase requirement of inotropic drugs in children with sepsis. Complete health facilities are able to detect troponin levels to evaluate the therapeutic use of vasoactive and inotropic drugs. However, troponin levels assessment is not possible in healthcare units with limited facilities. Vasoactive-inotropic score (VIS), on the other hand, is a simpler assessment method that can also be used as an indicator for myocardial dysfunction due to its parallel growth with troponin levels.

The results of this study are consistent with the study previously done to assess troponin I level as the prognostic marker in pediatric patients treated in intensive care unit. The increase in troponin I in sepsis was the secondary result of cytokine-mediated myocardial depression or hypoperfusion or both cases that occurred together. Troponin was associated with VIS. The median of VIS was 0 (ranged

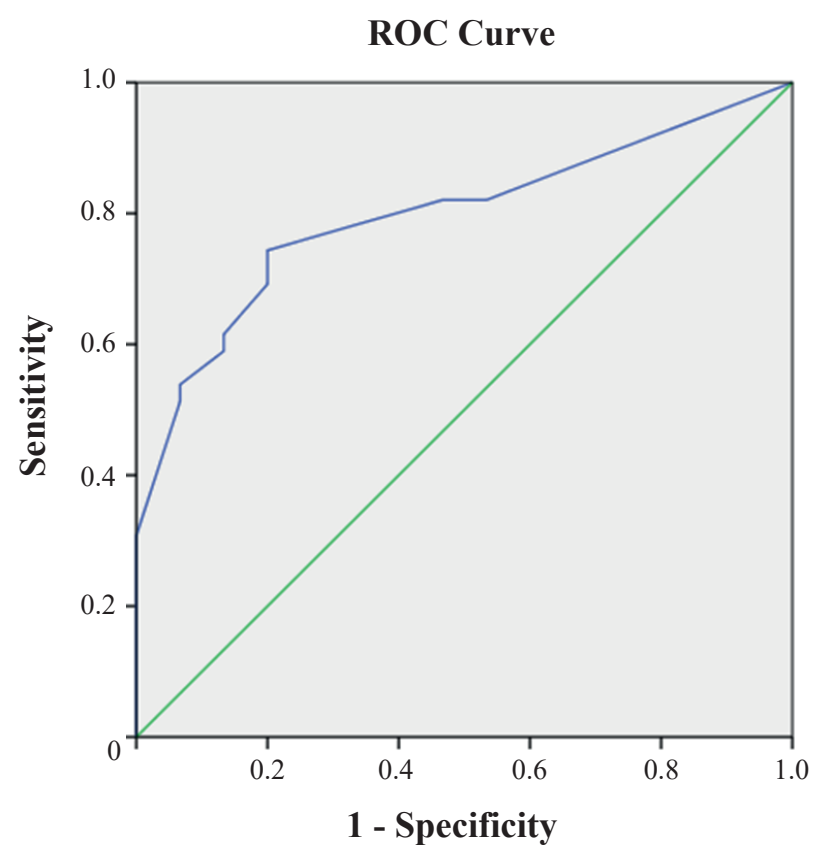

Figure 1. ROC curve of VIS at 48 hours. (AUC: $79.7 \%, p<0.001$, 95\% CI: 67.7-91.6\%). Diagonal segments are produced by ties. between 0 and 10) whereas troponin was not detected in samples. Meanwhile, VIS value was 16.5 (ranged between 0 and 52) when troponin level increased.(16)

Another study done had examined VIS biomarkers and myocardial dysfunction in children with sepsis. The long-term study found higher VIS values on the first day of treatment in non-survivors than in survivors $(63.0 \pm 16.4$ vs. $36.9 \pm 19.4 ; p<0.001)$. VIS continued to increase until the third day, from $37 \pm 14$ on the first day to $68 \pm 49$ on the third day. VIS decreased only on the seventh day to $17 \pm 29$ $(p<0.01)$. The decrease in creatine-kinase MB (CK-MB) as the myocardial dysfunction biomarker was accompanied by the decrease in VIS $(p=0.04)$ and PELOD-2 scores $(p<0.01) .(15)$

In this study, VIS at 48 hours and the peak during treatment were observed to be associated with the mortality of sepsis in children ( $p=0.001$ and $p<0.001$, respectively). In non-survivors, VIS at 48 hours and the peak during treatment had higher scores than the scores of the survivors. ROC curve, with $79.7 \%$ AUC, showed that at 48 hours measurement, VIS value could be a predictor of mortality in children with sepsis $(p<0.001 ; 95 \%$ CI $67.7 \%-91.6 \%)$. A past retrospective study done to 90 children in PICU with septic shock stated that pediatric risk of mortality (PRISM) III, sequential organ failure assessment (SOFA) and VIS scores described the severity of the disease. VIS values in non-surviving patients were higher than the survivors (mean \pm SD 56.68 $\pm 58.88 ; 27.66 \pm 38.46$ respectively).(17) Similarly another study done in in Turkey using medical record of 93 children with septic shock reported significant differences in maximal inotropic scores between patients who survived and not in the first 24 hours and 48 hours $(p<0.05)$. The cut-off inotropic score was at 15 , with $69.76 \%$ sensitivity and $50.98 \%$ specificity. Inotropic scores at 24 hours and 48 hours can be independent predictors of mortality in critically ill pediatric patients with septic shock. (18) VIS value at 48 hours in this study can predict mortality if it is equal or more than 11 with $74.4 \%$ sensitivity and $80 \%$ specificity. Children with sepsis having VIS 48 hours cut-off at $\geq 11$ were at 1.99 times greater risk of mortality $(95 \% \mathrm{CI}$ 1.25-3.19) than patients with VIS value less than 11. 
All past studies about VIS in children have only associated VIS with cardiac surgery in pediatrics. No study has investigated the correlation between VIS and troponin as a biomarker for myocardial dysfunction (MD) in sepsis. Besides that, the needs of vasoactive and inotropic drugs can also serve as the predictor of mortality in children. This study has shown that VIS at 48 hours can be used as an indicator of MD to evaluate pediatric therapy with sepsis, especially in health units with limited facilities. It also provided the cut-off values of VIS at 48 hours to predict the mortality of sepsis in children.

\section{Conclusion}

The VIS at 48 hours can be an alternative to troponin level assessment to evaluate suitable vasoactive and inotropic therapy, as well as to predict mortality in children with sepsis. This is an advantage, especially in healthcare units with limited laboratory facilities, to early detect myocardial dysfunction in children with sepsis. VIS value at 48 hours has a positive correlation with troponin $\mathrm{T}$ and troponin $\mathrm{I}$ in children with sepsis. It can also serve as a prognostic factor of mortality with cutoff point of 11 , in which patients with VIS score $\geq 11$ were at 1.99 times greater risk of mortality than patients with $<11$ VIS score.

\section{Authors Contribution}

$\mathrm{ACD}$ and $\mathrm{ADL}$ were involved in concepting and planning the research, ACD performed the data acquisition/collection, calculated the experimental data and performed the analysis, ACD and ADL drafted the manuscript and designed the figures. ACD, ADL and ML took parts in giving critical revision of the manuscript.

\section{References}

1. Dezouza DC, Barreira ER, Faria LS. The epidemiology of sepsis in childhood. Shock. 2017; 47: 2-5.

2. Hassan B, Morsy S, Siam A, Ali AS, Ando M, Shafie MA, et al. Myocardial injury in critically ill children: a case-control study. ISRN Cardiol. 2014; 14: 919150. doi: 10.1155/2014/919150.
3. Wu JR, Chen IC, Dai ZK, Hung JF, Hsu JH. Early elevated B-type natriuretic peptide levels are associated with cardiac dysfunction and poor clinical outcome in pediatric septic patients. Acta Cardiol Sin. 2015; 31: 485-93.

4. Jambagi M, Shetty R. Fluid therapy and hemodynamic support in septic shock. J Pediatr Crit Care. 2018; 5: 56-66.

5. Samsu N, Sargowo D. Sensitivitas dan spesifisitas troponin T dan I pada diagnosis infark miokard akut. Majalah Kedokteran Indononesia. 2007; 57: 363-72.

6. Rosjo H, Varpula M, Hagve TA, Karlsson S, Ruokonen E, Pettila V, et al. Circulating high sensitivity troponin $\mathrm{T}$ in severe sepsis and septic shock; distribution, associated factors, and relation to outcome. Intensive Care Med. 2011; 37: 77-85.

7. Dauhan AC, Lubis AD, Mutiara E, Lubis M. Correlation of troponin level (troponin T, troponin I) with PELOD-2 score in sepsis as a predictive factor of mortality. Open Access Maced J Med Sci. 2019; 7: 4072-77.

8. Misango D, Pattnaik R, Baker T, Dunser MW, Dondorp AM, Schultz MJ, et al. Haemodynamic assessment and support in sepsis and septic shock in resource-limited settings. Trans R Soc Trop Med Hyg. 2017; 111: 483-9. doi: 10.1093/trstmh/try007.

9. McIntosh AM, Tong S, Deakyne SJ, Davidson JA, Scott HF Validation of the vasoactive-inotropic score in pediatric sepsis. Pediatr Crit Care Med. 2017; 18: 750-7.

10. Gaies MG, Jeffries HE, Niebler RA, Pasquali SK, Donohue JE, Yu S, et al. Vasoactive-inotropic score is associated with outcome after infant cardiac surgery; an analysis from the pediatric cardiac critical care consortium and virtual PICU system registries. Ped Crit Care Med. 2014; 15: 529-37.

11. Weiss L, Fitzgerald JC, Pappachan J, Wheeler D, JaramilloBustamante JC, Salloo A, et al. Global epidemiology of pediatric severe sepsis: the sepsis prevalence, outcomes, and therapies study. Am J Respir Crit Care Med. 2015; 190: 1147-57.

12. Marlies O, Salma A, Emma T, Jessica L, Katie L, John S. Cardiac troponin release is associated with biomarkers of inflammation and ventricular dilatation during critical illness. Shock. 2017; 47: 702-8.

13. Kakihana Y, Ito T, Nakahara M, Yamaguchi K, Yasuda T. Sepsis induced myocardial dysfunction: pathophysiology and management. J Intensive Care. 2016; 4: 22-9.

14. Rudiger A, Singer M. Mechanism of sepsis induced cardiac dysfunction. Crit Care Med. 2007; 35 (6): 1599-608.

15. Baranwal AK, Deepthi G, Rohit MK, Jayashree M, Angurana SK, Kumar MP. Longitudinal study of CPK-MB and echocardiographic measures of myocardial dysfunction in pediatric sepsis: are patients with shock different from those without? Indian J Crit Care Med. 2020; 24: 109-15.

16. Wilson C, Sambandamoorthy G, Holloway P, Ramnarayan P, Inwald DP. Admission plasma troponin I is associated with mortality in pediatric intensive care. Pediatr Crit Care Med. 2016; 17: 831-36.

17. Choi SJ, Ha EJ, Jhang WK, Park SJ. Platelets indices as predictive markers of prognosis in pediatric septic shock patients. Iran $\mathrm{J}$ Pediatr. 2017; 27: e7212. doi: 10.5812/ijp.7212.

18. Sevketoglu E, Anil A, Kazanci S, Yesilbas O, Akyol M, Bayraktar S, et al. Is an inotrope score a predictor of mortality and morbidity in children with septic shock? Crit Care. 2015; 19 (Supp 1): 147. 